Modeling the Wason Selection Task: A Response to Ragni and Johnson-Laird (2020)

\author{
David Kellen \\ Syracuse University \\ Karl Christoph Klauer \\ Albert-Ludwigs-Universität Freiburg
}

Author Note

David Kellen, Department of Psychology. Karl Christoph Klauer, Department of Social Psychology and Methodology. We thank Scott D. Brown and Edgar Erdfelder for their valuable comments. David Kellen received support from the Swiss National Science Foundation Grant 100014_165591. Karl Christoph Klauer was supported by DFG Reinhart-Koselleck grant DFG Kl 614/39-1.

Correspondence should be sent to David Kellen (davekellen@gmail.com). 


\begin{abstract}
Kellen and Klauer (this issue) discussed a number of limitations in Ragni, Kola, and Johnson-Laird's (2018) evaluation of theories of the Wason Selection Task. In a reply, Ragni and Johnson-Laird (this issue) raise a number of counter-arguments along with new arguments against the Inference-Guessing Theory. We argue that much of their reply hinges on a number of conceptual and methodological confusions as well as a mischaracterization of some of our main points.
\end{abstract}

Keywords: hypothesis testing, mental models, reasoning, selection task 
Modeling the Wason Selection Task: A Response to Ragni and Johnson-Laird (2020)

Kellen and Klauer (this issue) argued that the meta-analysis by Ragni, Kola, and Johnson-Laird (2018) comparing theories of the Wason Selection Task was inconclusive and lacked diagnostic value in several respects. In their reply, Ragni and Johnson-Laird (this issue, henceforth referred to as RJ-L) not only defended their meta-analysis, but also provided new arguments for the superiority of the mental-model account (MT) over the inference-guessing theory (IGT; Klauer, Stahl, \& Erdfelder, 2007). For this reason, it is necessary for us to not only address RJ-L's defense of their meta-analysis, but also their new criticism of the IGT model.

\section{Canonical Selections}

One of Ragni et al. (2018)'s comparisons focused on the prediction of 4 out of the 16 possible card selection patterns in the Wason selection task, which they refer to as "canonical". According to Ragni et al., twelve out of sixteen candidate theories of the selection task can be rejected due to their inability to predict all of the canonical selections. We criticized the designation of these four patterns as "canonical" and the dismissal of the remaining twelve patterns as "idiosyncratic and rare" (Ragni et al., 2018, p. 784). Specifically, we argued that the focus on any subset of data needs to be motivated by the structural properties of the models being compared (e.g., Birnbaum, 2008; Kellen \& Klauer, 2015), and not by a frequency criterion that is: ${ }^{1}$

(i) Inconsistently Applied: Ragni et al.'s (2018) criterion was to exclude any selection occurring less than $\frac{1}{16}$ of the times. However, Ragni et al. did not follow the criterion that they themselves established, as that would require for the "partial insight" selection $p q \bar{q}$ to be excluded. Otherwise, one would also have to include $q$ and $p \bar{p} q \bar{q}$, as they occur more frequently overall (see Table 2 in Ragni et al. 2018).

\footnotetext{
${ }^{1}$ To be clear, our reference to these patterns as "canonical" is nothing more than shorthand.
} 
(ii) Historically Biased: Ignores the fact that many studies were designed with the specific purpose of increasing the occurrence of certain selections such as p $\bar{q}$ (e.g., Cheng \& Holyoak, 1985; Platt \& Griggs, 1993).

(iii) Narrow-Minded: Dismisses experimental studies showing a targeted influence over selections such as q, p̀q, or $\bar{p} \bar{q}$ (e.g., Evans \& Lynch, 1973; Gigerenzer \& Hug, 1992; Stahl, Klauer, \& Erdfelder, 2008).

(iv) Unfair: Competing theories are not on equal footing when it comes to their ability to attribute canonical selections to a guessing process.

In their response, RJ-L defend the exclusion of selections such as q, stating that "as Ragni et al. argued, good theoretical grounds exist for rejecting these selections as idiosyncratic in the standard selection task" (p. 7). Unfortunately, we failed to identify the passages in Ragni et al. (2018) that detail these good theoretical grounds. In any event, when the endeavour is to pit theories against each other on the grounds of the selections that they can or cannot predict, it seems inappropriate to rule out a priori the selections that one's preferred theory cannot predict.

RJ-L also defended their inclusion of the partial-insight selection pq $\bar{q}$ as a canonical pattern, pointing out it "became the most frequent of all in Wason's (1969) study" (p. 8). Clearly, RJ-L are not proposing a general amendment to their criterion. If that were the case, then the hundreds of published studies on the Wason selection task would require the inclusion of many other selection patterns. Instead, RJ-L are calling for the Wason (1969) study to be granted a special, definitory role. So let us take a closer look at Wason's (1969) study: Across five trials, participants were requested to select from the same set of four cards. Prior to each new selection, the experimenter pointed out increasingly more directly and explicitly that it is necessary to also select the $\bar{q}$-card. In their initial selection trial, the partial-insight pattern pq $\bar{q}$ occurred for 2 of 32 participants, whereas the most frequent selections were $\mathrm{p}(16 / 32)$ and pq (13/32). In subsequent trials, $\overline{\mathrm{q}}$ was added to many 
selections, leading to a greater occurrence of $p \bar{q}$, and $p q \bar{q}$, and to a lesser extent $p \bar{p} q \bar{q}$, all at the expense of patterns not involving the $\bar{q}$-card. Based on the study procedure, it seems clear that the observed prevalence of pq $\bar{q}$ in later selections can be largely attributed to the nudged addition of the $\overline{\mathrm{q}}$-card to previous pq selections. Although interesting, it is not clear why this nudging manipulation should be granted some special status relative to all the other Wason selection task studies.

RJ-L were also unfazed by the existence of experimental manipulations, however robust, reliable, and/or theoretically motivated, that affect the selection of specific non-canonical selections such as q. According to RJ-L, they are to be summarily dismissed, given that "a manipulation that increases the frequency of this hitherto negligible [selection of the $q$ card] or non-existent selection may show only that experiments can stupidify their participants." (p. 8), and that "there is no need to try to explain noise" (p. 18). RJ-L appear to be taking a rather peculiar stance, according to which the only studies that should be taken seriously are those that affect the selections that they deem "not stupid" or "not noise" (e.g., pq̄̄ in Wason, 1969). Unfortunately, RJ-L do not explain how this stance can coexist with the general goal of characterizing selection behavior in the Wason task, especially when dealing with theories that provide a principled account for so-called "stupid" or "noise" selections.

Along with their defense, RJ-L introduce a new distinction between face-to-face (presumably laboratory studies) and online studies. They argue that there is more "diversity" (in terms of lower statistical information as quantified by Shannon's measure) in the latter than in the former (p. 8). The implication here appears to be that only the canonical patterns occur more than $\frac{1}{16}$ of the times in the face-to-face/laboratory studies. First, it is important to keep in mind that the unit of analysis here is the study, and that any conclusion based on ten online studies can only be made with considerable caution. But perhaps more importantly, it is unclear how a possible greater diversity of selection behavior in online studies is to explain that pq $\bar{q}$ occurs less often than the non-canonical 
patterns $q$ and $p \bar{p} q \bar{q}$. After all, one should expect pq $\bar{q}$ to remain among the most common ones. Altogether, dismissing online data post-hoc from the meta-analysis, once they are seen to contradict one's theoretical preferences, requires much more careful and substantial justification than the one provided by RJ-L.

When concluding their discussion on canonical selections, RJ-L state that

No need exists to change the canonical set. Moreover, the addition of new selections to it cannot rehabilitate any theories that fail to predict the four original ones. The set is viable, and its corroboration refutes 12 of the alternative theories. (p. 9)

This statement is inconsistent with their earlier acknowledgment that participants sometimes may guess in selecting cards and that

guessing can yield a canonical selection, too. Some way to correct for this problem is needed to assess the fit of a theory to the data (p. 8)

We agree with the latter statement. In fact, one of our main criticisms of Ragni et al.'s (2018) meta-analysis was that when guessing is taken into account, all of the twelve refuted theories are likely able to account for the rare partial-insight pattern via guessing (as done by the IGT). At the very least, the burden of proof over the claim that guessing cannot save these theories should lie with RJ-L. To be clear, our proposal is not to revise the canonical selections, but to consider all selections as a whole..$^{2}$ Our arguments for using the whole data are described in detail in Kellen and Klauer (this issue), so we will not repeat them here.

\footnotetext{
2 At multiple points, RJ-L allude to the idea that we were proposing changes to the set of canonical selections (e.g., p. 7 and p. 9). This is inaccurate. It may be that this misunderstanding comes from our argument that, if Ragni et al. (2018) were consistent in their application of their frequency criterion, then they would have ended with a very different set of canonical selections.
} 


\section{Dependencies in Card Selections}

Ragni et al. (2018) also evaluated theories based on their ability to capture the dependencies in card selection that are found in the data corpus. They rejected a total of nine theories out of twelve that predict independent selections. Kellen and Klauer (this issue) raised the well-documented point that one cannot infer dependencies at the individual-person level based on aggregate data (e.g., Davis-Stober \& Regenwetter, 2019; Estes, 1956; Estes \& Maddox, 2005; Kellen, Singmann, \& Batchelder, 2018; Regenwetter \& Robinson, 2017). Simply put, it is entirely possible to observe dependencies in the aggregate data even though each person selected cards independently. The only requirement is for people to not be identical in their selection probabilities.

It turns out that RJ-L (this issue) were not convinced by our tutorial explanation of this relationship between the aggregate and the individual probability distributions. Specifically, they claim that their evaluation is immune to the problems we raised, given that they quantified card-selection dependencies using Shannon's information measure $H: 3$

$$
H=-\sum_{i} P_{i} \log _{2}\left(P_{i}\right),
$$

with $P_{i}$ denoting the probability of observing the $i$ th selection pattern, with $i=1, \ldots, 16$.

In their original analyses, Ragni et al. (2018) showed that the informativeness of independent card selections is expected to much higher than what is observed in the aggregate data in their corpus. Now, RJ-L provide supplementary results showing that when splitting the experimental data into two arbitrary subsets at random 10,000 times,

\footnotetext{
${ }^{3}$ RJ-L's reaction denotes a gross misreading of the point we originally made. They begin by providing a definition of (in)dependence: two events $A$ and $B$ are independent if and only if $P(A \mid B)=P(A \mid$ not $B)$. They then proceed to state that we illustrate our claim by reporting a significant correlation, and that their approach has nothing to do with correlations. Much could be said about their argument, but we will limit ourselves to two clarifications: 1) we did not make any mention of 'correlations'. 2) What we did was directly test independence as stated above. The independence of two events implies that a $2 \times 2$ table describing their joint frequency can be accounted for by two parameters (e.g., $P(A)$ and $P(B)$ ). Our results showed that our artificial data example could not be accounted for by these two parameters.
} 
"in none of these splits did both subsets yield H's larger than their respective simulations based on independent selections." (p. 11). RJ-L do not explain how any of their simulations address our specific concerns. Nor could they, given that the problem at hand has nothing to do with the particular measure of dependence being adopted, and everything to do with the fact that there are strong constraints on what one can legitimately infer from aggregate data.

In an attempt to dispel any remaining confusions on this matter, let us provide a new example demonstrating that any given dataset may have arisen from individually independent selections. Consider the case that every participant in the population of participants responds with the selection pattern that would be observed for him or her, if included in the study sample, with probability 1 . This is a case of stochastically independent individual card selections, and the mixture of these independent participant-wise distributions trivially results in the distribution underlying the aggregate data, regardless of whether the information measure $H$ is high or low and whether card selections in the aggregate data are stochastically dependent or not. Although this is a somewhat artificial scenario, it is not difficult to construct more realistic examples (see Kellen and Klauer, this issue).

\section{Responses to Specific Comments on Model Fitting and Comparison}

1. RJ-L highlight the fact that we ended up fitting MT to the canonical-selection data corpus after we observed that the IGT is untestable and the parameters of the MT are not identifiable. We fail to see how RJ-L's point denotes any kind of inconsistency on our part or even qualifies any of our criticisms. After all, parameter estimation and model testing are orthogonal issues: Models can testable even when their parameters are not identifiable (e.g., Regenwetter \& Davis-Stober, 2012), and parameters can be estimated in conditions where models are not testable (e.g., Kellen

\& Klauer, 2018). Also, the evaluation of a model's ability to fit data (e.g., MT) does 
not depend on the testability of any other competing model (e.g., IGT; for an example and discussion, see Gelman \& Shalizi, 2013).

2. RJ-L argue that their goodness-of-fit results favoring MT are not impossible but in fact legitimate outputs of a well-known numerical optimization method, which turn out to not favor the IGT (p. 17). Let us reiterate our original point: The IGT can perfectly fit any pattern of canonical selections. This fact forces us to conclude that no model can fit the data better as there is no such thing as a better-than-perfect fit. This is especially clear when comparing the IGT and MT given that the latter misfits $28 \%$ of the datasets. Whether or not the values originally reported by Ragni et al. (2018) were produced by a known numerical algorithm (even if we ignore the MT misfits) is irrelevant, the same way that the equality $\frac{0.3}{3}=0.1$ is true regardless of the fact that the statement $' 0.3 / 3==0.1$ ' has output FALSE in every computer language that we know of. Ragni et al.'s (2018) fit results cannot be used to make any kind of case against the IGT — the only thing that they show is their failure to find the best IGT fits.

3. RJ-L point out that, despite our criticisms regarding Ragni et al.'s (2018) use of BIC, MT was still found to be more parsimonious than IGT (p. 17). We see ourselves forced to ask: So what? This in no way vindicates Ragni et al.'s use of BIC, which illegitimately over-penalized the IGT by arbitrarily retaining a non-identifiable parameter that made no contribution whatsoever.

4. More broadly, RJ-L's comments on model flexibility seem to be predicated on a number of misconceptions. One of these misconceptions is that the preference for any given model can be based on their parsimony alone. Even if quantified accurately, parsimony is only one of many attributes to be considered when evaluating models, and not by itself sufficient for establishing the superiority of a model over another (e.g., Myung \& Pitt, 2018; Kellen, 2019). Another misconception is that every 
parameter in a model amounts to an admission of ignorance (p. 19), rather than an acknowledgment of the different processes at play. Take an example from physics: the five parameters of the Ideal Gas Law, $P \cdot V=n \cdot R \cdot T$, do not reflect ignorance on the theorist's part but a precise knowledge of how different attributes such as temperature $T$ jointly determine the state of a gaseous body. We see no reason to think that parameters play a fundamentally different role in psychology (for a related discussion, see Kellen, Davis-Stober, Dunn, \& Kalish, 2020). As discussed below, the IGT parameters reflect a specific understanding of the different reasoning and guessing processes that has been experimentally validated. ${ }^{4}$

5. RJ-L also appear to assume that the number of parameters in a model is a good proxy for its flexibility. This understanding is evidenced by their reference to John Von Neumann's famous claim that he was able to fit an elephant with three parameters, and wiggle its trunk with four (Dyson, 2004). As humorous as Von Neumann's quote might be, it would be unreasonable to suggest that it constitutes a principled approach to model flexibility (for a recent review, see Myung \& Pitt, 2018). The idea that a model with many parameters is necessarily very flexible is refuted by the many counter-examples found in the literature at large (e.g., Regenwetter \& Davis-Stober, 2012). Also inaccurate is the notion that a model with very few parameters is necessarily parsimonious, as shown by Piantadosi's (2018) demonstration that a single parameter is always enough, even when there is wiggling involved.

6. The MT's misfits reported by Kellen and Klauer (this issue) show a systematic residual pattern, with the model overestimating pq and underestimating both $\mathrm{p}$ and pq $\bar{q}$. Using simulations, we show that these residuals are representative of the specific data patterns that MT cannot account for. In their response, RJ-L suggest that this underestimation might provide further support for the notion that pq $\bar{q}$ selections

\footnotetext{
4 The same cannot be said of MT, given that its parameters are not identifiable (see Kellen \& Klauer, this issue).
} 
reflect the inferential processes proposed by MT (p. 17). Surely there is a misunderstanding here, as we don't see how one could possibly extract such an ontological insight from the trivial fact that a model can only fail in the ways that it can fail (to be clear, our discussion of fit residuals was part of an investigation on how MT could fail). As a simple example, consider a binomial model assuming the order constraint $\theta \leq \frac{1}{2}$. It is easy to see that this model can only misfit data when the observed proportions are larger than $\frac{1}{2}$. In these cases, the predicted proportions will always be smaller than the observed ones.

\section{The New Arguments Against the IGT Model}

The focus of Kellen and Klauer (this issue) was the conclusiveness of Ragni et al.'s (2018) meta-analysis rather than making a case for the IGT. Because of this, we did not explain the IGT and its empirical support in much detail, relying on readers interested in it to turn to the original literature employing the model (Klauer et al., 2007; Stahl, Klauer, \& Erdfelder, 2008). But given that a considerable portion of RJ-L's comment is dedicated to criticize the IGT, we think that it is reasonable to address these criticisms here in all brevity.

RJ-L frequently complain about the numbers of parameters used in the IGT model. Ten parameters are used to account for sixteen selection frequencies. They suggest at different places that this makes it trivial that the model fits as well as it does; "With many free parameters, it becomes all too easy to fit erroneous theories to data." (p. 19). These and similar remarks fail to acknowledge that the IGT model prevails in a model-selection comparison with a number of models, some of them with more parameters (Klauer et al., 2007). RJ-L also fail to acknowledge the series of validation studies reported. Each study implemented experimental manipulations thought to influence one of the parameters, or only a few of them, while leaving the others unaffected.

For example, one of the parameters, $c$, describes the probability that the conditional rule is interpreted conditionally rather than biconditionally. Different manipulations 
believed to affect this aspect of participants' interpretation of the rule strongly affected the estimated value of parameter $c$ as expected (Exps. 1 to 4, Klauer et al., 2007). Moreover, experiments designed to affect other parameters did not affect this parameter's estimates (Exps. 5 and 6). Note in particular, that Klauer et al. (2007) successfully validated the IGT's four guessing parameters this way. Despite RJ-L's concerns, what these studies show is that each of the parameters is needed to succinctly capture the complex effects of the experimental manipulations on selection behavior as whole.

These experiments make the IGT model more than an exercise in model fitting: They underline the need of each of the used parameter in accounting for the data and actually turn an expression of ignorance into one of a validated measure or dependent variable: Inasmuch as each parameter is validated in a selective-influence study, it then becomes a valid measure of the process it is assumed to reflect (e.g., Batchelder \& Alexander, 2013). In other words, the estimated value of, for example, parameter $c$ tells us something about the proportion of participants who interpreted the rule conditionally rather than biconditionally. We acknowledge, however, that additional empirical work can and should be done to validate the different model parameters in this manner.

Furthermore, RJ-L highlight at several points that Kellen and Klauer (this issue) stated that the IGT "is purposely vague about the nature of the underlying reasoning process" (p. 6). RJ-L appear to interpret this as meaning that we are vague about the nature of the interpretation and reasoning steps participants are assumed to go through in the Wason selection task. However, a reading of Klauer et al. (2007) reveals that this is not the sense in which the model remains vague. Instead, the IGT is vague in the sense that it is not committed as to whether the interpretational and inferential steps are implemented in terms of mental-model algorithms (Evans, 1993), abstract reasoning rules (Braine \& O'Brien 1991), or linguistic processes (Polk \& Newell, 1995). What this means is that the IGT, by being formulated at a functional level (Marr, 1982), does not stand or fall with any of these theories. 
RJ-L also claim that the IGT does not take the meaning of the conditionals into account sufficiently, being based on formal rules of inference. This is difficult to understand given (i) that most of the parameters of the IGT model specify how participants interpret the meaning of the conditional rule, whereas (ii) Klauer et al. (2007) were purposely vague about whether the reasoning in the IGT model was couched in a semantic mental-models framework or a system of formal inference rules.

In any event, RJ-L acknowledge that the selection task cannot discriminate between formal rules of inference and meaning-based approaches, but they claim that the repeated Wason selection task can. Specifically, they cite a study by Johnson-Laird and Wason (1970) according to which participants switch to an exhaustive search for counterexamples once they have found "examples of a hypothesis", presumably the hypothesis specified by the conditional rule. Johnson-Laird and Wason (1970) described an experiment that has some resemblance to a Wason selection task involving multiple selections, in which participants experience that the selection of the q-card is non-diagnostic for assessing the truth of the hypothesis under scrutiny. Such learning can readily be handled by interpretational processes embodied in the IGT: The information helps clarify the meaning of the hypothesis - it is conditional rather than biconditional, which means that $\mathrm{p}$ is sufficient but not necessary for q. The learning experiences thereby make it unambiguously clear that informative inferences arise from having $\bar{q}$ as a premise, but not from $q$, leading to the selection of the former and the dismissal of the latter.

\section{Final Thoughts}

The central preoccupation of this reply was to dispel the many conceptual and methodological confusions that seem to populate Ragni and Johnson-Laird's arguments. Much to our frustration, we often found ourselves correcting mischaracterizations of our original arguments. Although most of RJ-L's arguments turn out to be pretty much untenable, we would like to highlight a silver lining, namely that Ragni et al. (2018) and 
RJ-L nevertheless point researchers towards a kind of approach that can be quite fruitful, if conducted appropriately: Construct a body of benchmark effects shown to be robust and reliable, and determine how well each theory is able to account for them. In this spirit, it would be interesting to see whether Wason's (1969) repeated-selection results can be replicated under conditions in which experimenter influence is ruled out. Moreover, a number of effects such as those observed in the negations paradigm (Evans \& Lynch, 1973) or those found in connection with perspective changes in thematic Wason tasks (Gigerenzer \& Hug, 1992) are also likely candidates (see also Klauer et al., 2007). If the studies from the Wason selection task have taught us anything, it is that we should not only search for instances that confirm our preferred theory, but also - and perhaps more eagerly — for counterexamples. 


\section{References}

Birnbaum, M. H. (2008). New paradoxes of risky decision making. Psychological Review, $115,463-501$.

Braine, M. D., \& O'Brien, D. P. (1991). A theory of if: A lexical entry, reasoning program, and pragmatic principles. Psychological review, 98, 182-203.

Cheng, P. W., \& Holyoak, K. J. (1985). Pragmatic reasoning schemas. Cognitive Psychology, 17, 391-416.

Davis-Stober, C. P., \& Regenwetter, M. (2019). The 'paradox'of converging evidence. Psychological review, 126, 865-879.

Dyson, F. (2004). A meeting with Enrico Fermi. Nature, 427, 297.

Estes, W. K. (1956). The problem of inference from curves based on group data. Psychological Bulletin, 53, 134-140.

Estes, W. K., \& Maddox, W. T. (2005). Risks of drawing inferences about cognitive processes from model fits to individual versus average performance. Psychonomic Bulletin \&3 Review, 12, 403-408.

Evans, J. S. B. (1993). The mental model theory of conditional reasoning: Critical appraisal and revision. Cognition, 48, 1-20.

Evans, J. S. B. T., \& Lynch, J. S. (1973). Matching bias in the selection task. British Journal of Psychology, 64, 391-397. doi: 10.1111/j.2044-8295.1973.tb01365.x

Gelman, A., \& Shalizi, C. R. (2013). Philosophy and the practice of bayesian statistics. British Journal of Mathematical and Statistical Psychology, 66, 8-38.

Gigerenzer, G., \& Hug, K. (1992). Domain-specific reasoning: Social contracts, cheating, and perspective change. Cognition, 43, 127-171. doi: 10.1016/0010-0277(92)90060-U

Johnson-Laird, P. N., \& Wason, P. C. (1970). Insight into a logical relation. Quarterly Journal of Experimental Psychology, 22, 49-61.

Kellen, D. (2019). A model hierarchy for psychological science. Computational Brain \& Behavior, 2(3-4), 160-165. 
Kellen, D., Davis-Stober, C. P., Dunn, J. C., \& Kalish, M. L. (2020). The problem of coordination and the pursuit of structural constraints in psychology. Retrieved from https://psyarxiv.com/3eupv

Kellen, D., \& Klauer, K. C. (2015). Signal detection and threshold modeling of confidence-rating ROCs: A critical test with minimal assumptions. Psychological Review, 122, 542-557.

Kellen, D., \& Klauer, K. C. (2018). Elementary signal detection and threshold theory. In E. J. Wagenmakers (Ed.), Stevens' Handbook of Experimental Psychology and Cognitive neuroscience (4th edition, vol. v). New York: Wiley.

Kellen, D., \& Klauer, K. C. (this issue). Theories of the wason selection task: A critical assessment of boundaries and benchmarks. Computational Brain $\&$ Behavior.

Kellen, D., Singmann, H., \& Batchelder, W. H. (2018). Classic-probability accounts of mirrored (quantum-like) order effects in human judgments. Decision, 5, 323-338.

Klauer, K. C., Stahl, C., \& Erdfelder, E. (2007). The abstract selection task: New data and an almost comprehensive model. Journal of Experimental Psychology: Learning, Memory, and Cognition, 33, 680-703.

Marr, D. (1982). Vision: A computational investigation into the human representation and processing of visual information. San Francisco, CA: WH Freeman.

Myung, J. I., \& Pitt, M. A. (2018). Model comparison in psychology. In Stevens' handbook of experimental psychology and cognitive neuroscience (p. 1-34).

Piantadosi, S. T. (2018). One parameter is always enough. AIP Advances, 8, 095118.

Platt, R. D., \& Griggs, R. A. (1993). Facilitation in the abstract selection task: The effects of attentional and instructional factors. The Quarterly Journal of Experimental Psychology, 46(4), 591-613.

Polk, T. A., \& Newell, A. (1995). Deduction as verbal reasoning. Psychological Review, 102, 533-566.

Ragni, M., \& Johnson-Laird, P. N. (this issue). Explanation or modeling: A reply to 
Kellen and Klauer. Computational Brain \& Behavior.

Ragni, M., Kola, I., \& Johnson-Laird, P. N. (2018). On selecting evidence to test hypotheses: A theory of selection tasks. Psychological Bulletin, 144, 779-796.

Regenwetter, M., \& Davis-Stober, C. P. (2012). Behavioral variability of choices versus structural inconsistency of preferences. Psychological Review, 119, 408-416.

Regenwetter, M., \& Robinson, M. M. (2017). The construct-behavior gap in behavioral decision research: A challenge beyond replicability. Psychological Review, 124, 533-550.

Stahl, C., Klauer, K. C., \& Erdfelder, E. (2008). Matching bias is not eliminated by explicit negations. Thinking and Reasoning, 14, 281-303. doi: 10.1080/13546780802116807

Wason, P. C. (1969). Regression in reasoning? British Journal of Psychology, 60, 471-480. 